\title{
The Impact of Clinical Pharmacist Interventions on Drug and Antibiotic Prescribing in a Teaching Hospital in Cairo
}

\author{
Osama H. Mohamed Ibrahim ${ }^{1,2}$, Suleiman El-Sharif ${ }^{3}$ \\ ${ }^{1}$ Department of Pharmacy Practice, College of Pharmacy, University of Sharjah, Sharjah, UAE; ${ }^{2}$ Department of Clinical Pharmacy, \\ College of Pharmacy, Cairo University, Cairo, Egypt; ${ }^{3}$ Department of Pharmaceutics and Pharmacology, College of Pharmacy, Uni- \\ versity of Sharjah, Sharjah, UAE. \\ Email: oibrahim@sharjah.ac.ae
}

Received July $4^{\text {th }}$, 2012; revised August $13^{\text {th }}$, 2012; accepted September $7^{\text {th }}, 2012$

\begin{abstract}
Background: The present study was undertaken to investigate the patterns of drug and antibiotics prescribing in a teaching hospital in Cairo, Egypt. Aim: To determine the impact of interventions on such trends in an attempt to rationalize drug use. Method: 1200 prescriptions and patients' records covering the months of January to December, 2011. Prescribing patterns were analyzed using WHO guidelines with regard to prescribing, patient care and health facility indicators. The same parameters were again assessed after distributing antibiotic guidelines and holding workshops activities directed towards rational drug use. Results: The number of hospital visits resulting in a prescription was significantly reduced from $94 \%$ to $86 \%$ (P-value < 0.05 ) and in both cases none of the encounters contained a generic drug. The average number of drugs per encounter was 2.7 and did not decrease significantly after intervention. A significant reduction was achieved in the number of prescriptions with antibiotics whereas reduction in encounters with injectable drugs was not statistically significant. Penicillins was the most commonly prescribed class of antibiotics and amoxicillin was the most frequently prescribed antibiotic. A significant reduction was observed in both encounters with penicillin and the total of those with antibiotics. Analysis of prescriptions with antibiotics revealed that penicillins, cephalosporins and erythromycin comprised $94 \%$ and $97 \%$ of all antibiotics prescribed before and after interventions respectively. Conclusion: The present results clearly indicated that interventions including distribution of antibiotic guidelines and running workshops and seminars on rational drug use to prescribers can lead to significant improvement in prescribing behavior.
\end{abstract}

Keywords: Intervention; Clinical Pharmacist; Teaching Hospital; Antibiotics; Cairo

\section{Introduction}

A plethora of drug utilization studies focused on assessing patterns of drug prescribing [1-5] as a mean of pinpointing areas for improvement with the aim to rationalize drug use. The health threats that can be caused by improper prescribing cannot be overlooked. Improper prescribing can cause toxicity for patients and will be a waste of money and time. It can also cause therapeutic failure that results in progress of disease conditions and worsening of the patient health condition. The improper prescribing and excessive use of antibiotics can lead to loss of the effectiveness of currently used antibiotics [1-5]. The World Health Organization (WHO) has repeatedly emphasized the importance of drug utilization studies and developed indicators examine trends of prescribing and the health facilities [6]. Once irrational drug use in its various forms is determined, feasible means of intervention are tried with the hope to improve drug use [7]. The use of antibiotics has been the subject of several studies [8-10], because of the globally confirmed trends of under treatment, wrong antibiotic selection and the risk of consequent emergence of bacterial resistant [1,11]. Guidelines for rational prescribing have been developed [12] but unfortunately, they have neither been always available nor effectively implemented in many developing countries. However, drug utilization studies in Egypt are scarce and misuse of particularly antibiotics has not been extensively explored [13]. In the present study, we applied the WHO indicators to analyze the pattern of drug prescribing in a teaching hospital in Cairo, and studied patient care and health facility indicators. These indicators are summarized with their measured parameters before and after the study intervention in Table 1. During the period of the present study (2009), a clinical pharmacist, a clinical pharmacologist and a microbiologist formed the antibiotics control group of Kasr El Aini teaching hospital in Cairo. The group prepared guidelines for antibiotic use in the hospital that were circulated to 
Table 1. Results of drug-use indicators (according to WHO guidelines) before and after intervention.

\begin{tabular}{|c|c|c|}
\hline Drug-use indicator & Before & After \\
\hline \multicolumn{3}{|l|}{ Prescribing indicators } \\
\hline Average number of drugs per encounter & 2.7 & 2.5 \\
\hline$\%$ of drugs prescribed by generic name & 0 & 0 \\
\hline$\%$ of encounters with an antibiotics prescribed & 47 & $34^{*}$ \\
\hline$\%$ of encounters with an injection & 18 & 16 \\
\hline$\%$ of drugs prescribed from PHC drug formulary & 100 & 100 \\
\hline$\%$ of visits resulting in a prescription & 94 & $86^{*}$ \\
\hline \multicolumn{3}{|l|}{ Patient care indicators } \\
\hline Average consulting time (minutes) & 11.6 & 12.1 \\
\hline Average dispensing time (seconds) & 85 & 87 \\
\hline Percentage of drugs actually dispensed & 100 & 100 \\
\hline Percentage of drugs adequately labeled & 97 & 98 \\
\hline \multicolumn{3}{|l|}{ Health facility indicators } \\
\hline Availability of key drugs (\%) & 93 & 94 \\
\hline $\begin{array}{l}\text { Availability of a copy of an essential drugs } \\
\text { list or formulary (\%) }\end{array}$ & 100 & 100 \\
\hline
\end{tabular}

*Statistically significant, P-Value $<0.05$.

all practicing physicians within the hospital. Moreover, workshops were held concentrating on rational drug use. In the present study, we evaluated the impact of such interventions on the drug use particularly of antibiotics.

\section{Methods}

Twelve core indicators were applied as described by the WHO [6] and International Network for the Rational Use of Drugs (INRUD). These include prescribing indicators namely; average number of drugs per encounter, and percentage of visits resulting in a prescription, prescribed generics, encounters with antibiotics, injection and drugs prescribed from hospital drug formulary. An additional indicator, the percentage share of each antibiotic, i.e. the proportionate distribution of antibiotics prescribed, was included to identify the frequency of individual antibiotics prescribed. We also studied patient care indicators such as average consulting time, average dispensing time, and percentage of drugs actually dispensed and those adequately labeled. For the health facility indicators, we assessed the availability of drugs and a copy of an essential drug list or formulary.

The present study was carried out during the months January to December, 2010. For prescribing indicators we used data from patient's records and prescriptions. The total number of patients studied was 1200 . The average consultation time expressed in minutes was calculated by dividing the total time for a series of consultations by their number and the average dispensing time expressed in seconds was determined by dividing the total time for dispensing medications to patients by the total number of those patients. Interventions applied include distribution of generic antibiotic guidelines to physicians and running workshops and seminar activities aimed at increasing awareness to rational drug use. These interventions were implemented in June, 2012 thus information collected for the period of January to May covered data before interventions. The study was approved by the hospital ethical committee before the commencement of study.

For statistical analysis, SPSS (version 18) was used. Frequency distribution was obtained and the MannWhitney test was used for nonparametric data analysis to ascertain the significance of differences between pre-and post-intervention data. P-value of $<0.05$ was considered significant.

\section{Results}

Results of the study of prescribing indicators are shown in Table 1.

The number of hospital visits resulting in a prescription was significantly reduced from $94 \%$ to $86 \%$ and in both cases none of the encounters contained a generic drug. The reduction in the average number of drugs per encounter was not significant (2.7 to 2.5). A significant reduction was achieved in the number of prescriptions with antibiotics whereas reduction in encounters with injectable drugs was not statistically significant. The time of both consultation and medication dispensing remained the same before and after intervention and so the percentage of adequately labeled drugs. Penicillin was the most commonly prescribed class of antibiotics and amoxicillin was the most frequently prescribed drug. A significant reduction was observed in both encounters with penicillin and the total of those with antibiotics. Analysis of prescriptions with antibiotics revealed that penicillins, cephalosporins and erythromycin comprised $94 \%$ and $97 \%$ of all antibiotics prescribed before and after interventions respectively (Table 2). However, after interventions, the non-significant reduction in penicillin use was compensated for by double increase in cephalosporin prescribing and slight increase in erythromycin use.

\section{Discussion}

In the present study, the average number of drugs per encounter was 2.7 and was not significantly reduced after intervention. Such a number is larger than the optimal WHO figure of 2 drugs per encounter, less than those reported in Indonesia and Nigeria (3.3 and 3.8 respectively) [14] and far in excess of those described for United Arab Emirates, Bangladesh and Lebanon [1,15, 16]. It is rather intriguing that although all the drugs 
Table 2. Antibiotics prescribed as a percentage of the total drugs prescribed and as the percentage share of antibiotics prescribed before and after intervention.

\begin{tabular}{ccccc}
\hline \multirow{2}{*}{ Antibiotics } & \multicolumn{2}{c}{$\begin{array}{c}\text { Percentage } \\
\text { of total }\end{array}$} & \multicolumn{2}{c}{$\begin{array}{c}\text { Percentage share of } \\
\text { antibiotics }\end{array}$} \\
\cline { 2 - 5 } & Before & After & Before & After \\
\hline Penicillins & 37.5 & 26.8 & 78 & 70 \\
Cephalosporins & 4.5 & 5 & 8 & 16 \\
Erythromycin & 5.3 & 5.2 & 8 & 11 \\
Tetracyclines & 1 & 0 & 1.5 & 0.2 \\
Sulfonamides & 0.7 & 0.55 & 1.7 & 1.3 \\
Aminoglycosides & 0 & 0.15 & 1.3 & 0.8 \\
Chloramphenicol & 0.4 & 0.2 & 0.5 & 0.2 \\
Other antibiotics & 0.4 & 0.5 & 0.5 & 0.3 \\
Anti-tuberculosis & 0.1 & 0.1 & 0.5 & 0.2 \\
Total & 50.5 & 39.5 & 100 & 100 \\
\hline
\end{tabular}

prescribed were from the hospital available formulary that listed only generic drugs, all drugs were prescribed by their proprietary names. This may be attributed to the very effective drug promotional activities that target physicians. It seems important to include in the curriculum of medical schools and also in continuing medical educational programs material emphasizing the general aspects of essential drug list and generic prescribing. Slightly less than $50 \%$ of all encounters were for an antibiotic and such a prescribing trend was significantly reduced in prescription issued following the intervention. The high percentage of visits resulting in prescription was similarly influenced by the intervention. Such positive changes were not observed in the number of encounters with injectable drugs. Both the consultation and dispensing times were not significantly altered after the intervention. However, although the average consultation time (11.6 minutes) seems reasonable and comparable to figures reported for UAE [8] but it is longer than figures reported for Bangladesh, Indonesia and Nigeria [14]. On the other hand the dispensing time of 85 seconds seems rather short to explain to patients the various aspects on the safe use and storage of the prescribed medications. The obviously short dispensing time may be attributed to lack of a pharmacy technician and the high patientspharmacist ratio. All the prescribed drugs were actually dispensed and 97 of the drugs were adequately labeled before and intervention. The percentage availability of key drugs was 93 before and 94 after intervention and a copy of the formulary of drugs was always available during the period of the study.

A significant reduction in the percentage of total antibiotics prescribing from $50 \%$ to $39.5 \%$, and the most commonly prescribed antibiotics were penicillin with amoxicillin being on top of list as the most commonly prescribed of all penicillins. In the present study prescribing antibiotics was almost double that in UAE [1], comparable to that in Norway [17] and lower than that reported for Sudan, Iran and England [18-20]. Penicillins, cephalosporins and erythromycin comprised $94 \%$ and $97 \%$ of all antibiotics prescribed before and after interventions respectively (Table 2). However, after interventions, the non-significant reduction in penicillin use was compensated for by double increase in cephalosporin prescribing and slight increase in erythromycin use.

\section{Conclusion}

In conclusion, the present study clearly indicated that interventions including distribution of antibiotic guidelines and running workshops and seminars on rational drug use to prescribers can lead to significant improvement in prescribing of antibiotics. However, educational circulars and programmes on prescribing generic drugs are needed. This can be enforced through introducing such concepts and those of rational prescribing in curriculum of medical schools.

\section{Acknowledgements}

The author is very grateful for Dr. Amal Hussein and Dr. Ghada Mansour from Kasr El Aini hospital pharmacy for their help in data collection and prescriptions checking. The author is also grateful for Dr. Hanzada Mahboub for her help in reviewing the data analysis.

\section{REFERENCES}

[1] S. Sharif, M. Al-Shaqra, H .Hajjar, A. Shamout and L. Wess, "Patterns of Drug Prescribing in a Hospital in Dubai, United Arab Emirates,” Libyan Journal of Medicine, Vol. 3, No. 1, 2008, pp. 10-12.

[2] Y. M. Irshaid, M. Al Homrany, A. A. Hamdi, K. K. Adjepon-Yamoah and A. A. Mahfouz, "Compliance with Good Practice in Prescription Writing at Outpatient Clinic in Saudi Arabia,” Eastern Mediterranean Health Journal, Vol. 11, No. 5-6, 2005, pp. 922-928.

[3] E. Yousif, A. M. Ahmed, M. E. Abdalla and M. A. Abdelgadir, "Deficiencies in Medical Prescriptions in a Sudanese Hospital,” Eastern Mediterranean Health Journal, Vol. 12, No. 6, 2006, pp. 915-918.

[4] G. N. Hamadeh, L. M. Dickerson, B. R. Saab and S. C. Major, "Common Prescriptions in Ambulatory Care in Lebanon," The Annals of Pharmacotherapy, Vol. 35, No. 5, 2001, pp. 636-640. doi:10.1345/aph.10175

[5] T. M. Vijayakumar, D. Sathyavati, T. Subhashini, G. Sonika and M. D. Dhanaraju, “Assessment of Prescribing Trends and Rationality of Drug Prescribing," International Journal of Pharmacology, Vol. 7, No. 1, 2011, pp. 140-143. doi:10.3923/ijp.2011.140.143

[6] International Network for Rational Use of drugs and World Health Organization, "How to Investigate Drug 
Use in Health Facilities: Selected Drug Use IndicatorsEDM Research Series No. 007," World Health Organization, Geneva, 1993.

[7] H.V. Hogerzeil, G. J. Walker, A. O. Sallami and G. Fernando, "Impact of an Essential Drugs Programme on Availability and Rational Use of Drugs,” The Lancet, Vol. 1, No. 8630, 1989, pp. 141-142. doi:10.1016/S0140-6736(89)91152-5

[8] M. Y. Hasan, M. Das and F. Mourad, "Drug Utilization and Antibiotic Use in the Primary Health Care Centres in Sharjah,” Eastern Mediterranean Health Journal, Vol. 3, No. 3, 1997, pp. 444-451

[9] M. Kuyvenhoven, R. de Melker and K. Van der Velden, "Prescription of Antibiotics and Prescribers' Characteristics. A Study into Prescription of Antibiotics in Upper Respiratory Tract Infections in General Practice,” Family Practice, Vol. 10, No. 4, 1993, pp. 366-377. doi:10.1093/fampra/10.4.366

[10] C. M. Kunin, "Evaluation of Antibiotic Usage: A Comprehensive Look at Alternative Approaches," Review of Infectious Diseases, Vol. 3, No. 4, 1981, pp. 745-753.

[11] W. E. Farrar, "Antibiotics Resistance in Developing Countries," Journal of Infectious Diseases, Vol. 152, No. 6, 1985, pp. 1103-1106. doi:10.1093/infdis/152.6.1103

[12] J. J. Marr, H. L. Moffet and C. M. Kunin, "Guidelines for Improving the Use of Antimicrobial Agents in Hospitals: A Statement by the Infectious Diseases Society of America,” Journal of Infectious Diseases, Vol. 157, No. 5, 1988, pp. 869-876. doi:10.1093/infdis/157.5.869

[13] A. Zaki, M. Abdel-Fattah, A. Bassili, M. Arafa and R. Bedwani, "The Use of Medication in Infants in Alexandria, Egypt,” Eastern Mediterranean Health Journal, Vol.
5, No. 2, 1999, pp. 320-327.

[14] H. V. Hogerzeil, Bimo, D. Ross-Degnan, R. O. Laing, D. Ofori-Adjei, B. Santoso, A. K. Azad Chowdhury, A. M. Das, K. K. Kafle, A. F. Mabadeje, et al., "Field Tests for Rational Drug Use in Twelve Developing Countries,” The Lancet, Vol. 342, No. 8884, 1993, pp. 1408-1410. doi:10.1016/0140-6736(93)92760-Q

[15] A. B. Guyon, A. Barman, J. U..Ahmed, A. U. Ahmed and M. S. Alam, "A Baseline Survey on Use of Drugs at the Primary Health Care Level in Bangladesh," Bulletin of the World Health Organization, Vol. 72, No. 2, 1994, pp. 265-271.

[16] G. N. Hamadeh, L. M. Dickerson, B. R. Saab and S. C. Major, "Common Prescriptions in Ambulatory Care in Lebanon,” The Annals of Pharmacotherapy, Vol. 35, No. 5, 2001, pp. 636-640. doi:10.1345/aph.10175

[17] M. Lindbaek, D. Berild, J. Straand and P. Hjortdahl, “Influence of Prescription Patterns in General Practice on Anti-Microbial Resistance in Norway," British Journal of General Practice, Vol. 49, No. 443, 1999, pp. 436-440.

[18] W. J. Bannenberg, C. J. Forshaw, D. Fresle, A. O. Salami and H. A. Wahab, "Evaluation of the Nile Province Essential Drugs Project: Mission Report by a WHO Team, Sudan, 27 April-12 May, 1991,” WHO, Geneva, 1991.

[19] A. A. Moghadamnia, M. R. Mirbolooki and M. B. Aghili, "General Practitioner Prescribing Patterns in Babol City, Islamic Republic of Iran,” Eastern Mediterranean Health Journal, Vol. 8, No. 4-5, 2002, pp. 550-555.

[20] A. Majeed and K. Moser, “Age- and Sex-Specific Antibiotic Prescribing Patterns in General Practice in England and Wales in 1996," British Journal of General Practice, Vol. 49, No. 446, 1999, pp. 735-736. 